\title{
LA MECANIZACIÓN DEL CAMPO EN ANDALUCÍA SEGÚN LOS CENSOS AGRARIOS DE 1972, 1982 Y 1989
}

Abelardo VICO RUIZ*

"Si se halla en gran parte exento de los inconvenientes y peligros físicos a que se expone el obrero de fábrica el empleo de las máquinas en la agricultura, su tendencia a suprimir de su puesto al trabajador, se realiza en ella con mayor fuer$z a "$ (Marx, K. El Capital).

\section{INTRODUCCIÓN}

\subsection{Resumen}

Se ha intentado valorar la intensidad de la mecanización agrícola en Andalucía relacionándola con la presentada por el conjunto estatal. Los resultados parecen conclusivos: la mecanización -en este caso identificada con tractorización- del campo andaluz es escasa. Para explicar este hecho se barajan algunas causas: insuficiente capitalización, lo cual conduce a la preferencia o la necesidad del alquiler de maquinaria en lugar de la compra de ésta, baja especialización productiva, altos niveles de agricultura a tiempo parcial y escasa difusión del fenómeno. No obstante, este análisis general no se puede mantener para todo tipo de explotaciones. En aquellas empresas de grandes dimensiones (+100 Has.) Andalucía exhibe unas cotas de mecanización superiores a las observadas en España.

Por otra parte, los datos revelan cómo los índices de mecanización son sistemáticamente más elevados en las pequeñas explotaciones, descendiendo éstos a medida que se trata de explotaciones de mayores dimensiones. La explicación de este fenómeno no es económica, sino técnica.

Además del estudio de la tractorización se han apuntado algunas ideas sobre la situación de los motocultores y las cosechadoras de cereales en la agricultura andaluza. La presencia de ambos tipos de máquinas es realmente escasa.

* Licenciado en Geografía por la Universidad de Granada. 
Por último, las premisas de la nueva PAC no favorece en absoluto el aument del número de máquinas en el futuro inmediato. En su lugar, sí se puede agudiź un proceso que ya se ha iniciado según Naredo (1989), el incremento de la poter cia, la velocidad y la adaptabilidad de los aperos, sustituyendo cantidad por cal dad, e intentando adaptar la máquina a las exigencias de cada zona, suelo, labore: etc.

\subsection{Imposibilidad de encontrar un índice de mecanización total}

Medir el grado de mecanización con los datos estadísticos que ofrecen 1c Censos Agrarios -los únicos válidos en ocasiones como ésta, sobre todo por su nivє de agregación-, resulta una tarea casi imposible. Dichos datos no permiten real. zar una medición aceptable, cuando menos exacta, del nivel de mecanización tot: de una zona. Nos ofrecen dos opciones de medición, y ambas presentan notable carencias:

a) Si consideramos sólo el número total de máquinas o el número total de C' de potencia existentes por cada 100 Has. afectadas ${ }^{1}$, estaríamos obviando u aspecto capital como es el alquiler de maquinaria. No podemos afirmar qu el grado de mecanización total de una zona es superior al de otra, solament porque la cantidad de máquinas o de $\mathrm{CV}$ por cada 100 Has. sea superio Hemos de conocer además con qué intensidad se alquila la maquinaria, pue un área puede gozar de un escaso número de máquinas, pero utilizarlas d una manera muy intensa debido a una arraigada costumbre o necesidad d arrendamiento, con lo cual, presenta un campo tan mecanizado como otr que cuente con mayor número de máquinas, pero donde estas no se alquile de una forma tan profusa.

b) Si considerando sólo el número total de máquinas o de $\mathrm{CV}$, exclusivament atendemos a la maquinaria en propiedad y no tenemos en cuenta el alquile de ésta, busquemos una altemativa que permita aglutinar en una mism medición la mecanización en propiedad y la mecanización en alquiler. Est puede hallarse, por ejemplo, al dividir el número de explotaciones totale: Con ello obtendremos un índice, el cual delatará el grado de mecanizació total. Ahora bien, obtener el tanto por ciento de explotaciones que emplea maquinaria respecto al total, que es en definitiva en lo que consiste el ind

1. Al hablar de "afectas" nos referimos a las hectáreas afectadas por el fenómeno realizado por la máquir en cuestión. Es decir, si tratamos de tractores consideraremos Has. "labradas", si hablamos de cosechador: de cereales consideraremos Has. "sembradas de cereal" (en este caso se desconsiderarían girasoles y otrc cultivos industriales). El índice definido por $\mathrm{CV}$ de potencia por cada $100 \mathrm{Has}$. labradas es un índic generalizadamente aceptado para medir la tractorización. 
cador anterior, no es un índice que ofrezca demasiadas garantías. Podríamos decir que carece de la finura suficiente. Porque, al considerar que una explotación de 2.000 Has. utiliza maquinaria, no conocemos si la emplea en diez de esas hectáreas o en mil. Puede ocurrir que esa explotación de 2.000 Has. utilice maquinaria en diez hectáreas y otra del mismo tamaño la utilice en 1.900 , y sin embargo, ambas son valoradas de la misma manera y sin diferencias, cuando las diferencias son, como vemos, muy notables. Según esto, lo ideal sería poder saber cuántas hectáreas son mecanizadas, aún sólo aproximadamente. Este sí sería un baremo fino. No obstante, hoy no se considera necesario recopilar este dato, por lo tanto no se dispone de él.

Así, y en definitiva, el índice que puede ser hallado (a), no es aceptable, y aquel que sería aceptable (b), no puede ser hallado².

Ante esta problemática, hemos optado por hallar, por un lado los índices de mecanización en propiedad o basados en el número de $\mathrm{CV}$ existentes, y por otro los índices de mecanización en alquiler. En ello basaremos nuestros análisis.

\subsection{Selección de las variables}

Hemos escogido como variable esencial el tractor. Por lo tanto, al hablar de mecanización en el presente trabajo estaremos tratando sensu estricto de tractorización. El papel de otras máquinas agrícolas como motocultores y cosechadoras de cereales, aún no siendo desdeñable, es sin duda menos importante. Es por ello que le dedicamos a ambas un pequeño comentario en el Apartado II.

\subsection{Medición de las variables}

Para proceder a la medición de la tractorización hemos utilizado el número total de CV de potencia existente y lo hemos dividido entre la cantidad de Has. afectadas por el fenómeno, en este caso el total de hectáreas labradas ${ }^{3}$. Con ello hemos

2. Con objeto de poder mejorar la información sobre la mecanización, resultaría adecuado introducir una pregunta más en el cuestionario del Censo Agrario. Así, no sólo se preguntaría al empresario qué tipo de maquinaria usa en la explotación, sino también cuántas hectáreas se ven afectadas aproximadamente por el trabajo de la máquina en cuestión. Conociendo este extremo podríamos delimitar de una manera más exacta el grado de mecanización total de un determinado territorio agrario.

3. El número total de CV se ha calculado del modo más simple, y quizás el único posible considerando los datos disponibles. Los Censos Agrarios nos ofrecen la cantidad de tractores por intervalos de CV. Así, el Censo de 1972 establece cuatro intervalos, menos de $25 \mathrm{CV}$, entre 25 y $60 \mathrm{CV}$, entre 60 y $80 \mathrm{CV}$ y más de 80 CV. Los Censos de 1982 y 1989 amplían la cantidad de intervalos y establecen cinco. A saber, menos de $34 \mathrm{CV}$, entre 34 y 54, entre 55 y 79, entre 80 y 108 y más de $108 \mathrm{CV}$. Para hallar el volumen de CV existente 
calculado la cantidad de CV por cada 100 Has. labradas. Esto en lo que se refiet al total de máquinas existentes. Para medir el alquiler de maquinaria no hemc tenido más remedio que recurrir a ese índice que, aunque algo burdo, es el únic que poseemos. Es un indicador menos fino que el empleado para medir la mec: nización en propiedad, pero al menos nos muestra las tendencias generales. As hemos dividido el número de explotaciones que usan maquinaria en alquiler ent1 el total de explotaciones.

En el caso de los motocultores se ha dividido la cantidad total de artefactos ent1 las hectáreas labradas. En este caso no se consideran CV de potencia -con los cualє se procedería sin duda a una medición más fina- porque los datos censales n desagregan los motocultores según su caballaje.

Para las cosechadoras se contempla el volumen total de máquinas -no se tí nen en cuenta los CV por la misma razón aducida por los motocultores- y el tot: de Has. sembradas de cereal.

\subsection{Justificación de la fecha de inicio del estudio}

Se ha elegido 1972 como fecha del inicio del estudio, porque ya existe un tr: bajo previo que analiza la mecanización del campo andaluz hasta esa fecha

\section{LA MECANIZACIÓN AGRÍCOLA EN ANDALUCÍA}

\subsection{La mecanización agrícola. Un tema olvidado por la geografía}

Al observar la bibliografía sobre geografía agraria de los últimos tiempos, : aprecia que el tratamiento de la mecanización agrícola es más bien parco. Fue méri de la profesora Ferrer Rodríguez (1978) -un mérito reconocido ya por Ortę Cantero (1983) - señalar el olvido del tema de la mecanización agrícola en el ser de la geografía. Hoy, quince años después de su estudio, persiste la laguna en es tema, pues los trabajos realizados han sido pocos y muy puntuales salvo exce] ciones.

en un intervalo multiplicamos el número total de tractores incluidos en él por el número medio de CV c intervalo. Por ejemplo, si en 1972 existían en Andalucía 100 tractores que tenían entre 25 y $60 \mathrm{C}$ multiplicaremos 100 por 42,5 , siendo 42,5 el resultado de la operación $25+6 / 2$. Para los intervalos abiertı el inferior y el superior, hemos escogido unos límites seleccionados según una premisa concreta, por deb o por encima de ese caballaje no suelen faricarse tractores al menos de manera regular. Como límite inferi de los intervalos menos de $25 \mathrm{CV}$ y menos de $34 \mathrm{CV}$ se ha estipulado $20 \mathrm{CV}$. Para los intervalos más de y más de $108 \mathrm{CV}$ se han fijado unos límites superiores de 120 y $180 \mathrm{CV}$ respectivainente.

4. FERRER RODRÍGUEZ, A. (1978). "La mecanización del campo en Andalucía". Cuadern Geográficos de la Universidad de Granada. Volumen del año 1978, págs. 117-129. Granada. 
Entre las causas de esta significativa amenesia hemos de eliminar a estas alturas una que barajaba como posible en su día la propia Amparo Ferrer. Probablemente las máquinas eran "entes de reciente implantación en el paisaje y no habían alcanzado en los esquemas metodológicos de las geografía clásica la categoría de «elementos integrantes»". Esto, que podía ser cierto hasta cierto punto en 1978 , indudablemente no lo es en 1993, por cuanto desde esa fecha a esa parte ha tenido lugar una introducción masiva de máquinas en el campo andaluz. En cuanto a la segunda y última argüida por la autora granadina, la existencia de unas fuentes "dispares, complejas e insuficientemente detalladas", la verdad es que tampoco la podríamos mantener tal cual. Y es que, aunque la información de los censos agrarios no haya mejorado excesivamente, sí se han denotado avances, y además, contamos con una variedad de fuentes notables según demuestra Seguellas Serrano en su trabajo (citado en bibliografía).

Por tanto, si por un lado las máquinas han alcanzado ya el estatus de elementos plenamente integrantes del paisaje agrario, y por otro las fuentes van mejorando, ¿cuáles son las verdaderas causas que explican el persistente abandono del tema por parte de la geografía?

Personalmente nos la consigo discemir. Ciertamente parece que los geógrafos consideramos la mecanización un tema residual. Señalar esto peca de ocioso por evidente a la vista de la bibliografía. Los argumentos -planteados desde la geografía por omisión, y desde otras disciplinas como la economía por acción- que atribuyen al camino técnico sólo un carácter residual, no resisten la crítica más elemental. Así lo demuestran autores como Schultz (1964) o Naredo (1971 y 1978), cuyas conclusiones son recogidas por Ortega (1983). A mi juicio, no hemos de olvidar que las modificaciones cuantitativas y cualitativas producidas en la mecanización han de entenderse como cambios acaecidos en uno de los medios de producción, el cual, como tal, tiene una cierta influencia en la configuración de la estructura agraria final. Sólo por ello debería desaparecer cualquier duda sobre su importancia y por lo tanto sobre la necesidad de su estudio.

El presente artículo pretende contribuir modestamente a subsanar el casi endémico abandono de la cuestión. En realidad se trata de un análisis de los niveles de mecanización del campo andaluz desde los primeros años setenta hasta el final de los ochenta, tomando como base los datos ofrecidos por los censos agrarios elaborados durante ese período. Estos datos censales deben ser entendidos con las limitaciones presentadas en el punto 1 de la introducción, sobre todo en lo referente a la mecanización en alquiler. 


\subsection{Escasa mecanización en el campo andaluz. Posible causalidad}

Resulta innecesario mencionar cómo para entender el valor real de unas simples cifras se han de poseer uno o varios puntos de referencia con las que compararlas. Es por ello que he creído conveniente tomar como sistemática referencia el conjunto estatal. Aunque, si bien es cierto que estos datos constituyen en todo momento una guía inequívoca, no lo es menos que encierran realidades agrarias muy dispares las cuales quedan enmascaradas al utilizarlos en su globalidad. Esto último es tan obvio como importante.

El volumen de maquinaria por unidad de superficie existente en Andalucía siempre ha sido inferior al ofrecido por España. Concretamente en 1989 la región andaluza alcanzaba los $150 \mathrm{CV} / 100$ Has. labradas, mientras que España se elevaba a los $231 \mathrm{CV} / 100$ Has. labradas. Además, las diferencias se han ido incrementando con el tiempo. La tractorización relativa ha aumentado en la Comunidad Autónoma Andaluza desde 1972 a 1989 un $164 \%$ frente al $233 \%$ estatal. Este retraso en la adquisición de tractores por parte de los agricultores andaluces ha de tener unas posibles causas, algunas de las cuales vamos a tratar de señalar:

a) Escasa capitalización-alquiler de maquinaria. La empresa media andaluza debe poseer una capitalización más escasa que la empresa media española. Es por ello que muchos empresarios andaluces, sobre todo pequeños y medianos, no disponen del capital suficiente para aventurarse en la fuerte inversión que supone la compra de un tractor. Y, como en los tiempos que corren la máquina se ha convertido en casi imprescindible, se ven obligados a alquilarla. Entre los empresarios andaluces se encuentra muy arraigado el alquiler de maquinaria (por ejemplo en 1989 Andalucía exhibía un índice de alquiler de 55 frente a 38 de España). Un alquiler que por lo demás aparece como la solución más sencilla y lógica a la dificultad o imposibilidad de compra. Resulta altamente significativo como el alquiler de maquinaria agrícola aparece extendido en Andalucía como en ninguna otra comunidad autónoma, según demuestra la oportuna comprobación.

b) La especialización productiva. Una empresa agraria que disfrute de un policultivo puede que posea más dificultades para aprovechar racionalmente los servicios mecanizados que otra decidida por el monocultivo o al menos por un policultivo menos variado e intenso. Según Ortega Cantero, Lebeau (1972) y Gilbank (1974) han demostrado las dificultades que para su mecanización encierra el policultivo, llegando a catalogar al mismo como "unc de los problemas fundamentales de la transformación tecnológica del espacio agrario" (Ortega Cantero, 1983). 
Admitiendo que esto es así, hemos de aceptar que, según los datos censales, la especialización ha sufrido un avance poco considerable en Andalucía. Tanto la superficie que reunía olivar-frutales-viñedo, como aquella que asociaba herbáceos con cualquier otro cultivo, ha descendido en España bastante más significativamente. La primera se ha reducido casi a la mitad entre 1982 y 1989 , mientras que la segunda lo ha hecho al menos en un cuarto en el conjunto estatal, sin embargo en el espacio andaluz apenas ha descendido la misma en un 27 y un 18 por ciento respectivamente. Pero no hemos de considerar sólo que las asociaciones de cultivos o el policultivo ha descendido poco en Andalucía, sino que ya de por sí el policultivo es más frecuente en Andalucía que en España. Según los datos del Censo Agrario de 1982, las asociaciones expuestas con antelación ocupan el 2,61\% de las tierras labradas en Andalucía, mientras que en España sólo constituyen el 2,15 \% de las mismas. Por tanto, según la evaluación de este rasgo las explotaciones andaluzas en conjunto, con su escasa tendencia a la especialización productiva, no han favorecido excesivamente la introducción de maquinaria propia.

c) La difusión de maquinaria. En 1979 Nguyev hablaba de la decisiva importancia de la difusión tecnológica para el aumento de la productividad. Al hilo de ello se podría plantear también la decisiva importancia de la difusión de los puntos de venta de tecnología para la compra de esa tecnología. Siguiendo esta pauta se ha tratado de encontrar un indicador sencillo del grado de difusión de la maquinaria agrícola tanto en Andalucía como en España. Calculado éste ha resultado que el índice de difusión ${ }^{5}$ de Andalucía ascendía al 9,0 mientras que el de España se elevaba a 14,1. Quiero esto decir que los agricultores andaluces en particular tienen el fenómeno más lejos especialmente. Así, esta menor cercanía se puede constituir en uno de los factores que les complique la adquisición de maquinaria.

d) La agricultura a tiempo parcial. Particularmente pienso que una dedicación parcial a la agricultura desestimula en muchos casos la adquisición de maquinaria propia. La ATP parece más intensa en Andalucía que en España.

5. Esta tasa de difusión responde al siguiente cálculo:

Número de trabajadores empleados en la "construcción de máquinas agrícolas y tractores agrícolas
Número de trabajadores empleados en el "comercio al por mayor de maquinaria agrícola"

Número de ocupados agrícolas

Al final leeremos, cantidad de personas relacionadas con la construcción y venta de maquinaria agrícola por cada 1.000 ocupados agrícolas. Los datos del numerador han sido extraídos del Censo de Locales de 1980, mientras que los del denominador corresponden al Censo de Población de 1981. No ha resultado posible realizar el mismo cálculo para 1990, pues el Censo de Locales de este año no recoge los datos específicos sobre el comercio de maquinaria agrícola, sino sobre el comercio de maquinaria en general. 
En 1982 -datos del Censo Agrario, como es costumbre- los empresario agrarios cuya dedicación principal no era la agricultura se situaba en 1 . comunidad autónoma andaluza en el $74,8 \%$, frente a tan sólo un $66,1 \%$ el España. En 1989 la medición del fenómeno que permitía el Censo Agrariı era otra. Las jornadas completas medias trabajadas en la explotación por e propio titular de la misma eran en Andalucía de 34,6, una cantidad baja qu delata la menor dedicación del empresario andaluz a su explotación, sobri todo si consideramos que las jornadas completas medias trabajadas por e empresario español superan las 40 . No olvidemos en atención a esto qu ciertas investigaciones demuestran como la agricultura a tiempo parcial e más intensa en las zonas de más atraso económico. Por consiguiente, 1 , importancia de la agricultura a tiempo parcial en Andalucía puede ser en tendida como una de las circunstancias que explican la escasa adquisició 1 de maquinaria agrícola en la región.

\subsection{Mayor mecanización relativa de las pequeñas explotaciones respecto a las grandes. ¿Explicación económica o simplemente técnica?}

El análisis general expuesto hasta aquí puede y debe ser completado con u1 estudio del fenómeno según el tamaño de las explotaciones. En Andalucía -al igua que en España- los mayores índices de mecanización corresponden a las explota ciones pequeñas (0-10 Has.) mediano-pequeñas (10-15 Has.), y así mismo est índice decae a medida que las propias explotaciones aumentan de tamaño.

Cuando observamos que las pequeñas explotaciones poseen en término medi más maquinaria que las grandes, podríamos deducir de ello que su capitalizacióı o renta relativa es así mismo superior, pues ésta sería una forma de explicar pre cisamente su mayor adquisición de maquinaria. Este punto resultaría extremada mente llamativo de entrada, y más aún cuando conocemos como diversos investi gadores insisten en las dificultades económicas de las pequeñas explotaciones. $\mathrm{S}$ ir más lejos, Terrafeta mantiene que las explotaciones menores de $20 \mathrm{Has}$. y sobrı todo las inferiores a 5 sufren una "inviabilidad teórica". El mismo defiende qu muchas pequeñas explotaciones pueden ser muy rentables, pero su escasa dimen sión no les permite generar unas rentas suficientes (Тепrafeta, 1979). Por tanto, nı creo certero pensar que la capitalización de las pequeñas explotaciones sea supe rior a la presentada por las grandes, con lo cual, la causa de su más elevada me canización no puede radicar en esa mayor capitalización pues ésta parece irreal De este modo, posiblemente la razón deba ser buscada en otro sitio.

Pensemos por un momento en las variedades de explotaciones según su tamañ، y en las variedades de tractores según su tamaño. Sin atisbo de dudas las varieda des de explotaciones son mucho más numerosas que las variedades de tractores 
De esta manera, probablemente el gestor de una muy pequeña explotación tendrá más dificultades que el gran empresario para comprar el tractor, cuya potencia, según una lógica matemática, sea la que le corresponde al tamaño de su explotación, pues seguramente no existirá. En virtud de esas lógica matemática, si a una explotación de 100 Has. labradas le corresponde un tractor de $100 \mathrm{CV}$ de potencia, a una explotación de $1 \mathrm{Ha}$. labrada le corresponderá un tractor cuyo caballaje sea de $1 \mathrm{CV}$. No obstante, éste último no existe sino en las tiendas de juguetes. Así, ese empresario deberá comprar un tractor que exista, al menos de la mínima potencia fabricada $(+20 \mathrm{CV})$. Pero por lo demás, aún existiendo tractores de 1 $\mathrm{CV}$, el empresario tendrá que comprar al final aquél de 20 ó $25 \mathrm{CV}$, pues será a partir de esa potencia cuando verá garantizada la realización de sus labores agrícolas.

En este orden de cosas, un empresario que posee una explotación de $1 \mathrm{Ha}$. labrada y desea adquirir un tractor y no alquilarlo, adquiere un tractor de $20 \mathrm{CV}$ por ejemplo. Por otro lado, el dueño de una gran explotación de 100 Has. labradas compra un tractor de $100 \mathrm{CV}$. Con ello, mientras que la pequeña explotación posee un índice de mecanización de $20 \mathrm{CV} / \mathrm{Ha}$. labrada -tengamos en cuenta que se ha presentado un caso extremo-, la gran explotación se sitúa en $1 \mathrm{CV} / \mathrm{Ha}$. labrada. Bien es verdad que encontraremos muchos empresarios pequeños que no compran un tractor, pero la actuación de sólo uno de ellos compensa la abstinencia de compra de otros muchos colegas, pues como vemos, las diferencias del ejemplo (20 a 1) permiten más de una abstinencia.

$\mathrm{Si}$ en vez de tomar como índice CV/Has. labradas, elegimos tractores/Has. labradas, el hecho anterior se confirmará de una manera aún más rotunda por razones obvias. Con los mismos datos expuestos con antelación, a la explotación pequeña le correspondería un índice de 1 tractor/Ha. labrada, en cambio la gran explotación sólo alcanzaría un índice de 0,01 tractor/Ha. labrada (diferencisa de 100 a 1).

De este modo, si aceptamos esta hipótesis, aceptamos que la razón que explica la mayor mecanización de las pequeñas explotaciones no reside en la mayor capitalización de las mismas, sino en su escasa dimensión, o en la patente inadecuación entre su tamaño y el tamaño de los tractores. La causa es, como vemos, eminentemente técnica y no económica.

\subsection{Escasa racionalidad empresarial de las pequeñas explotaciones en el tema de la mecanización}

Por otra parte, en un estudio económico realizado sobre algunas comarcas andaluzas en 1984 se afirmaba que, en general, la compra de maquinaria a cargo de un pequeño empresario agrario suponía una disminución de sus rentas (Braulio Medel, director, 1984). Como hemos visto, los pequeños empresarios adquieren un 
gran volumen de maquinaria, ciertamente más que los grandes gerentes. Es por ello que podemos intuir como una compra de maquinaria importante por su parte provocará una también importante reducción de sus rentas. Esta disminución de rentas, de confirmarse, nos permitiría hablar de la escasa racionalidad empresarial existente en las pequeñas y pequeño-medianas explotaciones.

En virtud de esto último y un poco colateralmente, nos podemos preguntar si esta escasa racionalidad de los pequeños empresarios es buscada o impuesta en el tema de la mecanización. Es decir, si la mayor parte de las pequeñas explotaciones, con la sola compra de un tractor, realizan ya un desembolso el cual es inamortizable por definición manteniendo el nivel de beneficios previo a la compra del mismo, o si por el contrario, la compra de un tractor es un hecho económico que, como en las grandes explotaciones, se puede salvar con una adecuada gestión. Me inclino por la primera premisa y creo que la escasa racionalidad económica de las pequeñas explotaciones en el tema de la mecanización es una cuestión casi impuesta merced a la escasa capacidad de reacción económica que les permite su escasa dimensión.

\subsection{Comparación de las explotaciones andaluzas según su tamaño con las explotaciones españolas}

Una vez comprobado que las explotaciones pequeñas exhiben unos índices de mecanización superiores a aquellas de grandes dimensiones, pasemos ahora a comparar la mecanización de las pequeñas explotaciones andaluzas con la mecanización de las pequeñas explotaciones españolas, procediendo del mismo modo con las grandes explotaciones. Con ello pretendemos entrever la verdadera situación tanto de las pequeñas como de las grandes explotaciones andaluzas.

Las pequeñas explotaciones andaluzas, y aún las medianas aunque con menos intensidad, poseen un nivel de tractorización bastante más escaso al presentado por las pequeñas empresas españolas en general. Muy al contrario, las grandes explotaciones (+100 Has.) andaluzas disfrutan de una mecanización elevada, ciertamente superior a la mecanización del conjunto estatal. Por consiguiente, parece que las pequeñas y pequeño-medianas empresas agrarias andaluzas no resisten un análisis comparativo con sus homólogas españolas. Parece que los pequeños y medianos empresarios andaluces prefieren o se ven obligados a alquilar los tractores antes que a comprarlos. En cambio las grandes explotaciones de la comunidad autónoma andaluza sí que resisten ese análisis comparativo. Posiblemente estas grandes explotaciones andaluzas disfrutan de unas mejores condiciones, sobre todo presupuestarias, que las españolas, para la adquisición de maquinaria. 


\subsection{Evolución cronológica del número de tractores}

En otro orden de cosas, el aumento de tractores en el campo andaluz ha sufrido un parón en los últimos lustros. La subida vertiginosa acaecida en los años sesenta y setenta se ha visto contestada por una cierta ralentización en los ochenta. Este proceso se ha vivido también en España, incluso con más intensidad. El hecho fue ya advertido por Naredo (1989). "Hoy el proceso de mecanización -decía el veterano investigador" se encuentra próximo a la saturación en cuanto al número de tractores". Y es que, como él mismo indicaba seguidamente, "ya no se tiende a aumentar el número de tractores, sino su potencia, la velocidad de los mismos y la adaptabilidad de los aperos. Se ha pasado de la cantidad a la calidad, a intentar adaptar la máquina a las exigencias de cada zona, suelo, labores, etc.". Este hecho se puede comprobar fácilmente en el caso de la potencia. La potencia media de los tractores asciende en los años ochenta a un ritmo tan importante como el exhibido en los setenta, sin seguir en absoluto el parón experimentado por el número total de tractores.

\subsection{Constatación de los resultados por otros caminos}

Las conclusiones anteriores han sido extraídas basándonos exclusivamente en un índice, CV/Has. labradas. Por ello, alguien puede acusar al estudio de contemporizar, es decir, de buscar un índice que ofreciese unos resultados tales que rubricasen las hipótesis de partida, en definitiva, que se adaptase a nuestros propios requerimientos. Con el fin de evitar este extremo he procedido a obtener resultados mediante otros dos caminos:

1. Por medio del índice Tractores/Has. Iabradas. Con él se corrige la posible influencia desvirtuadora que puede causar el empleo de tractores cuyo caballaje fuese anormalmente extremado, bien por exceso o bien por defecto.

2. Por medio del porcentaje de tractores sobre el total que le corresponde a cada grupo de explotaciones de los considerados.

Mediante ambos caminos se han comprobado resultados similares si no equivalentes a aquellos derivados del índice CV/Has. labradas. Obsérvese ante todo la mayor y ascendente mecanización de las pequeñas explotaciones respecto a otras de grandes dimensiones, y las diferencias comparativas entre Andalucía y España, unas diferencias siempre favorables a España salvo en las grandes explotaciones. 


\section{CUADRO I \\ PORCENTAJE DE TRACTORES CORRESPONDIENTES A CADA TAMAÑO DE EXPLOTACIÓN}

\begin{tabular}{|c|c|c|c|c|c|}
\hline & & \multicolumn{4}{|c|}{ Tamaño explot. (Has.) } \\
\hline & & $0-10$ & $10-50$ & $50-100$ & +100 \\
\hline \multirow[t]{2}{*}{1972} & 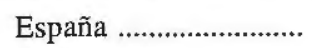 & 22,2 & 44,1 & 13,9 & 19,8 \\
\hline & Andalucía ..................... & 18,0 & 34,7 & 14,1 & 33,1 \\
\hline \multirow[t]{2}{*}{1982} & 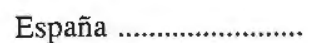 & 36,3 & 40,8 & 10,2 & 12,6 \\
\hline & Andalucía .................... & 26,6 & 35,8 & 11,4 & 26,1 \\
\hline \multirow[t]{2}{*}{1989} & 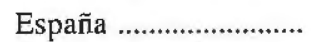 & 39,3 & 39,8 & 9,5 & 11,3 \\
\hline & Andalucía ...................... & 32,1 & 35,4 & 10,1 & 22,4 \\
\hline
\end{tabular}

Fuente: Elaboración propia a partir de los censos agrarios correspondientes.

\section{UN PEQUEÑO COMENTARIO SOBRE MOTOCULTORES Y COSECHADORAS DE CEREALES}

Los motocultores son poco importantes en Andalucía y se alquilan con poca frecuencia, pues no es una máquina que se adaptase a las características de la mayor parte de la superficie agraria de la región. Tengamos en cuenta sólo un aspecto, la parcelación. Sabemos que el motocultor es una máquina especialmente pensada para las parcelas pequeñas. En vista del Cuadro II deducimos que estas pequeñas parcelas no abundan excesivamente en Andalucía, de ahí la escasa proliferación de este tipo de máquinas.

\section{CUADRO II \\ NÚMERO MEDIO DE PARCELAS POR EXPLOTACIÓN}

\begin{tabular}{|c|c|c|c|}
\hline & 1972 & 1982 & 1989 \\
\hline 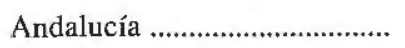 & 3,10 & 2,84 & 2,93 \\
\hline 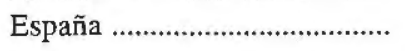 & 10,86 & 8,74 & 8,14 \\
\hline
\end{tabular}

Fuente: Elaboración propia a partir de los datos de los censos agrarios correspondientes.

Un análisis más pormenorizado según el tamaño de las explotaciones nos revela para la motocultorización signos prácticamente calcados de los observados en la tractorización. Así, la mecanización de las pequeñas explotaciones es frecuentemente elevada, descendiendo a medida que tratamos de explotaciones mayores. La 
explicación de este hecho no residirá casi con seguridad en la mayor capitalización de esas pequeñas explotaciones, sino en la inflación que provoca en los índices de mecanización la adquisición de un motocultor merced a su escasa dimensión -al igual que ocurre en los tractores-. Por otro lado, las pequeñas explotaciones andaluzas se encuentran bastante distanciadas en el valor de sus índices de sus homónimas españolas, no así las grandes explotaciones las cuales prácticamente utilizan casi tantos motocultores como las grandes explotaciones del conjunto del Estado.

En cuanto a las cosechadoras de cereales, su empleo se ha ido imponiendo de manera generalizada en el campo. No obstante, su elevado precio y las dificultades de amortización que conlleva su estrecha estacionalidad obligan a los agricultores a alquilarla cada vez con mayor asiduidad, renunciando por ello a alquilarla cada vez con mayor asiduidad, renunciando por ello a su compra. Hemos de considerar que el número total de máquinas ha descendido en la última década tanto en España como en Andalucía ${ }^{6}$. Sin embargo los niveles de alquiler han experimentado un ascenso espectacular. Si en 1972 sólo 17 de cada 100 explotaciones andaluzas alquilaban cosechadoras para la recogida, en 1989 se trataba ya de un $83 \%$ de las mismas. Tan espectacular aumento del arrendamiento no se aprecia ni en motocultores ni en tractores, pues su menor precio y las mayores facilidades de amortización que presentan -al ofrecer la posibilidad de emplearlos en un trabajo relativamente continuo a lo largo de todo el año- no hacen de la compra de los mismos un hecho tan inaccesible.

Descendamos a un análisis según el tamaño de las explotaciones. Las medianas y las grandes explotaciones han visto descender su índice de mecanización desde los años setenta, algo impensable en el caso de los tractores o de los motocultores. Lógicamente, ante la situación crítica del campo andaluz cada vez son más las empresas, por grandes que sean, que se encuentran en condiciones de realizar la inversión que supone la compra de una cosechadora de cereales. Además, la superficie cerealística ha ido descendiendo, con lo cual ésta se hacía menos rentable. Por ello estas medianas y grandes explotaciones han optado paulatinamente por el alquiler de las propias cosechadoras, con lo cual se han incrementado tan vertiginosamente dichos índices de alquiler en estas explotaciones.

Por otra parte, las pequeñas explotaciones exhiben unos índices de mecanización en cuanto a cosechadoras de cereales, por lo general mayores que las medianas o grandes empresas, sobre todo en el año 1989. Esta es una situación idéntica a la advertida en el caso de tractores y motocultores, y como en el mismo, posee equivalente explicación técnica -que no económica-.

6. Si el índice de mecanización de las cosechadoras de cereales asciende, no se debe, obviamente, al incremento del número de máquinas, sino al descenso considerable de la superficie sembrada de cereal. 


\section{EL FUTURO INMEDIATO DE LA MECANIZACIÓN EN ANDALUCÍA}

La nueva PAC diseñada por la Comisión a principios de los noventa estimula la extensificación, el abandono y el barbecho ${ }^{7}$. Debido a ello, la dimensión cultivada de muchas explotaciones descenderá provocando con ello una caída de su capitalización -a pesar de las ayudas a las rentas concedidas por los gobiemos-. Esta capitalización más baja y cuestiones como el propio aumento del barbecho hará desistir a muchos empresarios, sobre todo pequeños y medianos, en su intento de comprar maquinaria, obligándolos por lo demás a recurrir al arrendamiento de la misma. No sería de extrañar por tanto que en los próximos años asistiéramos a una cierta estabilización e incluso descenso en la adquisición de maquinaria, al menos en determinados tipos de explotaciones de cierto tamaño. Dicha disminución en la compra posiblemente será contestado con un aumento del alquiler de maquinaria. ante todo en las pequeñas y pequeño-medianas explotaciones. En este sentido, el análisis prospectivo de la mecanización para los próximos años no se presenta, en mi opinión, demasiado optimista.

Aunque, por otra parte, este posible descenso de la utilización de maquinaria puede provocar, junto a otros casi seguros descensos como el de fertilizantes, una cierta recuperación del balance energético hoy deficitario. Esta sería una verdadera señal reveladora de un cierto éxito a cargo de la agricultura biológica.

Por último me agradaría resaltar un hecho. Desde el punto de vista del tema de la mecanización resulta interesante establecer unos valores guías, los cuales, cada territorio según sus características particulares debería conseguir -si es que no se encuentra ya en ellos-. En el caso de la mecanización no podemos negar que constituye una tentación aventurar unos intervalos de mecanización idóneos o aconsejables para cada espacio. Pues bien, en mi modesta opinión no resultaría acertado

7. En mi opinión, el descenso continuado del barbecho durante la década de los setenta y ochenta se constituye en uno de los motivos que ha estimulado la compra de maquinaria. Por la misma regla de tres, un aumento del barbecho, si se produjerese, desestimularía dicha compra.

PORCENTAJE DE BARBECHO* (Barbecho/Tierras en cultivo)

\begin{tabular}{|c|c|c|}
\hline & Andalucía & España \\
\hline 1976 & 17,1 & 24,6 \\
\hline 1983 & 16,0 & 23,1 \\
\hline 1989 & 13,4 & 21,3 \\
\hline
\end{tabular}

Fuente: Elaboración propia a partir de los datos del Anuario de Estadística Agraria.

* En el Anuario de Estadísticas Agraria aparecen barbechos y otras tierras no ocupadas las cuales se definen como todas las tierras de cultivo en descanso o no ocupadas durante el año por cualquier motivo, aunque hayan sido aprovechadas como pastos para el ganado. Las cifras de este epígrafe han sido las utilizadas en el cuadro anterior. 
intentar establecerlos, por más que en geografía se haya hecho uso e incluso abuso de esta práctica en un intento de proponer esquemas de planificación, intentos por otra parte muy loables si se reconociesen ciertas limitaciones. Y es que, a la postre, "es delicado establecer como ideal o aconsejable un nivel de mecanización basado simplemente en una ratio que no admite la complejidad de la realidad física" (Braulio Medel, director, 1984).

\section{BIBLIOGRAFÍA}

FERRER RODRÍGUEZ, A. (1978): "La mecanización del campo en Andalucía". Cuadernos Geográficos de la Universidad de Granada, 8, págs. 117-129.

FIMA/83. "Conferencia Internacional de mecanización agraria". Zaragoza. Colaboran Centro Regional de Investigación y Desarrollo Agrario del Ebro y Colegio Oficial de Ingenieros Agrónomos de la Cuarta Región. Breve comentario de José María Fernández de Córdova, REAS, nº 124, pp. 133 (1983).

GARCÍA AZCÁRATE y otros (1985): "Mecanización agraria y empleo en el regadío extremeño". Revista de Estudio Agro-sociales, 132, págs. 173-191.

GARRABOU, R. (1990): "Sobre el atraso de la mecanización agraria en España (1850-1933)". Agricultura y Sociedad, 57, págs. 41-77.

MARTÍNEZ SERRANO, J.A. (1982): "La modernización del sector agrario", en Martínez Serrano, J.A. y otros. Economía Española 1960-1980. Crecimiento y cambio estructural. Madrid, H. Blume, págs. 55-79.

MEDEL CÁMARA, B., director (1984): Estudio económico de la comarca de Baza-GuadixHuéscar. Granada. Excma. Diputación Provincial de Granada.

ORTEGA CANTERO, N. (1983): "El proceso de mecanización y adaptación tecnológica del espacio agrario español". Agricultura y Sociedad, 27, págs. 81-149.

SARASA, J.L.A. (1988): "La mecanización de la agricultura en la región de Murcia". Revista de Estudios Agro-sociales, 147, págs. 155-170.

SEGUELLAS SERRANO, J.A. (1988): "Fuentes para el estudio de la mecanización agraria". Revista de Estudios Agro-sociales, $\mathrm{n}^{\circ} 146$.

TERRAFETA PUYOL, L. (1979): La capitalización de la agricultura española 1962-1975. Madrid. Blanco de Crédito Agrícola.

WOOD, R.H. (Jr) (1983): "Utilización de tractores y empleo en las grandes explotaciones privadas mexicanas". Revista Internacional del Trabajo, Vol. 102. Oficina Internacional del Trabajo. Ginebra. Comentario en REAS, nº 124, pp. 192 (1983). 